\title{
Study of the process $e^{+} e^{-} \rightarrow \pi^{+} \pi^{-} \pi^{0} \pi^{0}$ with SND at VEPP-2000
}

\section{Kharlamov Alexey*}

Budker Institute of Nuclear Physics, SB RAS, Novosibirsk, 630090, Russia Novosibirsk State University, Novosibirsk, 630090, Russia

E-mail: A.G.Kharlamov@inp.nsk.su

M. N. Achasov ${ }^{a, b}$, A. Yu. Barnyakov ${ }^{a}$, K. I. Beloborodov ${ }^{a, b}$, A. V. Berdyugin ${ }^{a}$,

A. G. Bogdanchikov ${ }^{a}$, A. A. Botov ${ }^{a}$, A. V. Vasilev ${ }^{a, b}$, V. B. Golubev ${ }^{a, b}$, K. A. Grevtsov ${ }^{a, b}$, T. V. Dimova ${ }^{a, b}$, V. P. Druzhinin ${ }^{a, b}$, I. M. Zemlyanskij ${ }^{a}$, L. V. Kardapoltsev ${ }^{a, b}$, A. G. Kharlamov ${ }^{a, b}$, D. P. Kovrizhin ${ }^{a}$, I. A. Koop ${ }^{a, b}$, A. A. Korol ${ }^{a, b}$, S. V. Koshuba ${ }^{a}$, A. S. Kupich ${ }^{a, b}$, A. P. Lysenko ${ }^{a}$, K. A. Martin ${ }^{a}$, A. E. Obrazovsky ${ }^{a}$, A. V. Otboev ${ }^{a}$, E. V. Pakhtusova ${ }^{a}$, E. A. Perevedentsev ${ }^{a}$, A. L. Romanov ${ }^{a}$, A. I. Senchenko ${ }^{a}$, S. I. Serednjakov ${ }^{a, b}$, P. Ju. Shatunov ${ }^{a}$, Ju. M. Shatunov ${ }^{a}$, D. A. Shtol ${ }^{a}$, D. B. Shvarts ${ }^{a}$. Z. K. Silagadze ${ }^{a, b}$, A. N. Skrinsky ${ }^{a}$, I. K. Surin ${ }^{a}$, Ju. A. Tikhonov ${ }^{a, b}$, Yu. V. Usov' ${ }^{a}$,

${ }^{a}$ Budker Institute of Nuclear Physics, Novosibirsk, 630090, Russia

${ }^{b}$ Novosibirsk State University, 630090 Novosibirsk, Russia

\begin{abstract}
Today the energy range between 1.4 and $2 \mathrm{GeV}$ gives a rather large error to hadron vacuum polarization (HVP) calculation, because the cross sections here are measured with insufficient accuracy mostly by ISR method. We present new high precision measurement of the $e^{+} e^{-} \rightarrow \pi^{+} \pi^{-} \pi^{0} \pi^{0}$ cross section with the SND detector at VEPP-2000 $e^{+} e^{-}$-collider. This process dominates in the energy region between 1 and $2 \mathrm{GeV}$ and determines the HVP error in this region. The percent level measurement will strongly influence $(g-2)_{\mu}$ interpretation and other precision SM tests. We have separated intermediate states of this reaction. The cross sections for the different intermediate states can be used to study properties of the $\rho(1450)$ and $\rho(1700)$ resonances.
\end{abstract}

XV International Conference on Hadron Spectroscopy-Hadron 2013

4-8 November 2013

Nara, Japan

\footnotetext{
* Speaker.
} 


\section{Introduction}

Today the energy range between 1.4 and $2 \mathrm{GeV}$ gives rather large error to hadron vacuum polarization(HVP) calculation, because the cross sections here are measured with accuracy $10 \%$ mostly by ISR method. We present new high precision cross section measurement of the process $e^{+} e^{-} \rightarrow \pi^{+} \pi^{-} \pi^{0} \pi^{0}$ with SND at VEPP-2000. This process dominates in the energy region between 1 and $2 \mathrm{GeV}$ and gives most part of the HVP error so the percent level measurement will strongly influence on $\mathrm{g}-2$ interpretation and other precision SM tests.

The cross section of the process $e^{+} e^{-} \rightarrow \pi^{+} \pi^{-} \pi^{0} \pi^{0}$ at energies $1 \mathrm{GeV}<\sqrt{s}<2 \mathrm{GeV}$ is determined by the transition of vector mesons $\mathrm{V}\left(\rho, \rho^{\prime}, \rho^{\prime \prime}\right)$ to the four $\pi$-mesons. The main intermediate states are $\omega \pi^{0}, a_{1} \pi, \rho \rho$ and $f_{0} \rho$.

\section{Experiment}

The Spherical Neutral Detector (SND) [1]is a general purpose non-magnetic detector. It was taking data from 2009 at the VEPP-2000 collider [2] in the energy range 360-2000 MeV. The detector includes several subsystems. There is a tracking system around beam pipe, based on multilayer jet-type drift chamber and one layer proportional chamber with charge division and cathode strip readout. Three layer electromagnetic calorimeter consists of $1632 \mathrm{NaI}(\mathrm{Tl})$ crystals and have $90 \%$ solid angle coverage. The energy resolution of the calorimeter for photons is $\sigma_{E} / E=$ $4.2 \% / \sqrt[4]{E(\mathrm{GeV})}$. The angular resolution is equal to $\sigma_{\phi}, \sigma_{\theta} \simeq 1.5^{\circ}$. Aerogel cherenkov counter between drift chamber and calorimeter is used as particle ID detector. The muon system includes scintillation counters and two layers of streamer tubes.

In our analysis, we used the statistics of two experiments, MHAD10 and MHAD11, collected in 2010-2011. The integrated luminosity in the MHAD10 and MHAD11 experiments was 5 and 25 $\mathrm{pb}^{-1}$, respectively. The integrated luminosity was measured by two independent methods: using events of electron-positron scattering and annihilation into two photons. The systematic uncertainty in the luminosity was estimated as the difference of these two measurements to be approximately $2 \%$.

\section{Event selection}

For our analysis, we selected events that satisfied the following conditions:

1. two or more charged tracks;

2. four or more reconstructed photons;

3. the distance from the beam axis to the event vertex in the R- $\phi$ plane is less than $1 \mathrm{~cm}$;

4. the coordinate of the event vertex along the beam axis is less than $10 \mathrm{~cm}$.

The conditions on the vertex coordinates are deter mined by the size of the interaction region, the drift chamber resolution and serve to suppress the beam background events and cosmic muons. 


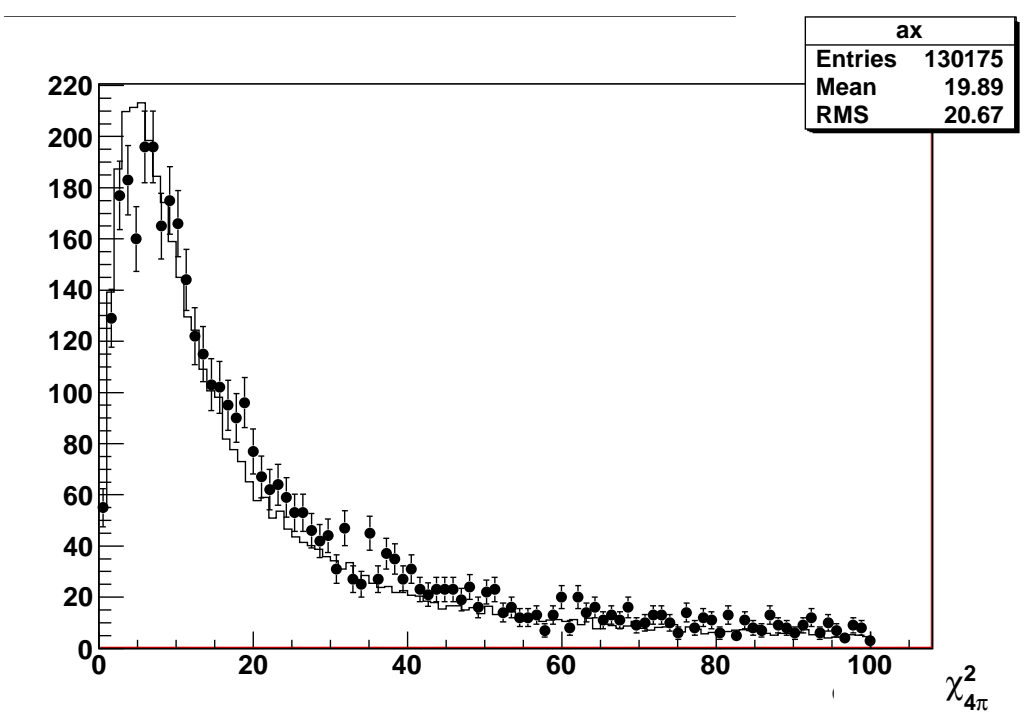

Figure 1: $\chi^{2}-$ distribution for kinematic fit in the hypotheses $e^{+} e^{-} \rightarrow \pi^{+} \pi^{-} \pi^{0} \pi^{0}$.

The longitudinal size of the interaction point depends on the beam energy; in the experiment, it changed from 2.0 to $2.5 \mathrm{~cm}$.

For the events selected under above conditions, we performed kinematic fits in the hypotheses: $e^{+} e^{-} \rightarrow$ $\pi^{+} \pi^{-} \pi^{0} \pi^{0}, e^{+} e^{-} \rightarrow \pi^{+} \pi^{-} \pi^{0}, e^{+} e^{-} \rightarrow \pi^{+} \pi^{-} \pi^{0} \gamma, e^{+} e^{-} \rightarrow \pi^{+} \pi^{-} 3 \pi^{0}, e^{+} e^{-} \rightarrow \pi^{+} \pi^{-} 4 \pi^{0}$.

After kinematic fit we used next cuts on likelihood value (Fig. 1): $\chi_{\pi^{+} \pi^{-} \pi^{0} \pi^{0}}^{2}<40, \chi_{\pi^{+} \pi^{-} \pi^{0}}^{2}>$ $20, \chi_{\pi^{+} \pi^{-} 3 \pi^{0}}^{2}>20, \chi_{\pi^{+} \pi^{-} 4 \pi^{0}}^{2}>20$.

\section{Cross-section}

The cross section of the process $e^{+} e^{-} \rightarrow \pi^{+} \pi^{-} \pi^{0} \pi^{0}$ at each energy point was determined using the formula:

$$
\sigma(E)=\frac{N_{4 \pi}(E)}{I L(E) \cdot \varepsilon(E)(1+\delta(E))},
$$

where $N_{4 \pi}$ is the number of events for the process under study, IL(E) is the integrated luminosity at a given point, and $\varepsilon(E)$ is the detection efficiency, $\delta(E)$ is radiative correction. The cross section is shown at Fig. 2.

Detection efficiency and radiative correction was determined by the Monte-Carlo method and slightly depends on energy. Mean value for detection efficiency for $a_{1} \pi$ intermediate state is $33.5 \pm$ $0.4 \%$ and for the $\omega \pi^{0}-32.5 \pm 1.4 \%$. Radiative correction is 0.12 at $1 \mathrm{GeV}$ and 0.14 at $2 \mathrm{GeV}$.

To obtain the correction factor for the detection efficiency we reconstruct events with lost track or lost photon. Correction factor was determined as

$$
\delta_{\pi, \gamma}=\frac{N_{4 \pi}}{\left.N_{4 \pi}+N_{l o s t \pi, \gamma}\right)} \cdot \frac{\varepsilon_{4 \pi+l o s t \pi, \gamma}(E)}{\varepsilon(E)}
$$




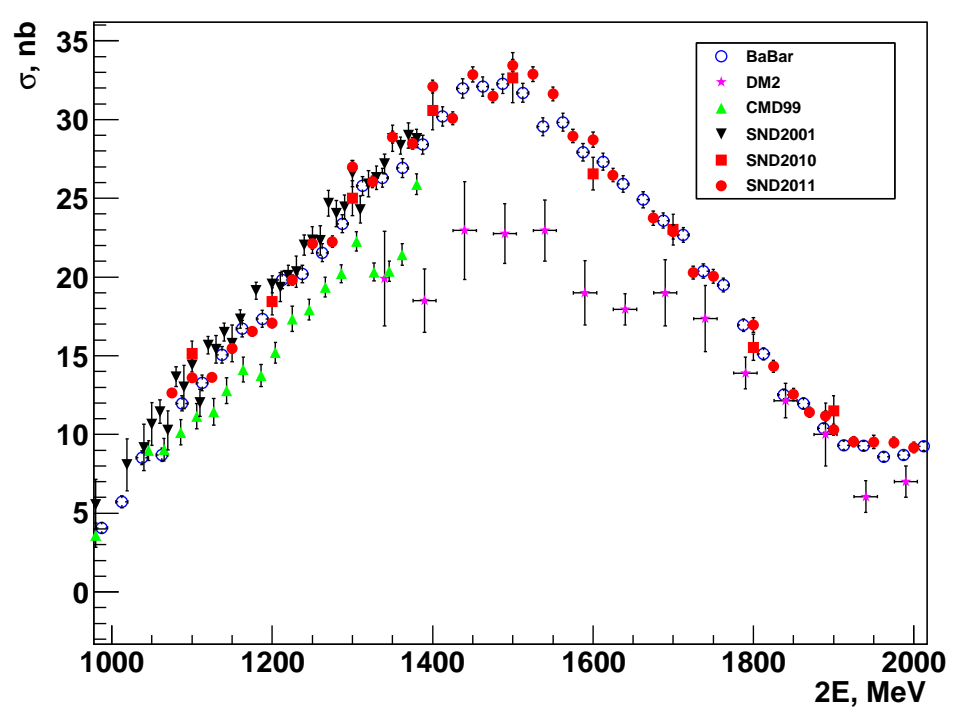

Figure 2: Cross section for the process $e^{+} e^{-} \rightarrow \pi^{+} \pi^{-} \pi^{0} \pi^{0}$.

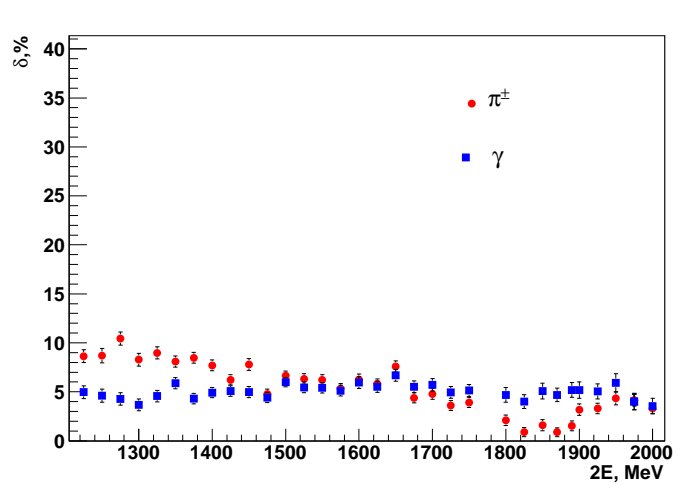

Figure 3: Efficiency correction.

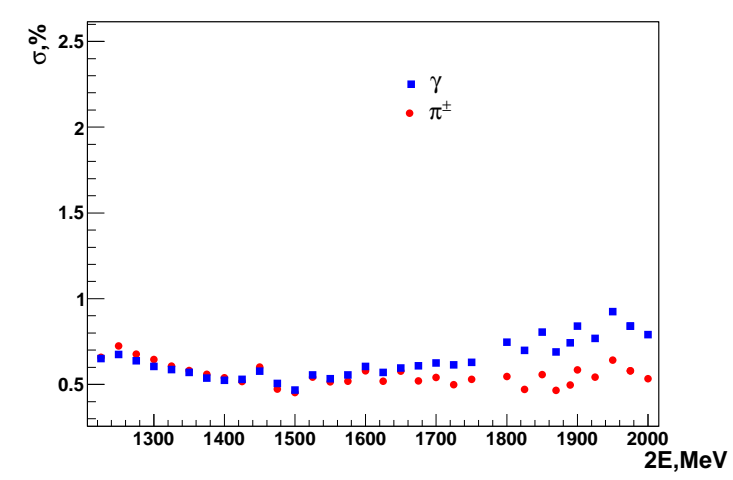

Figure 4: Efficiency correction. Uncertainty of the efficiency correction.

where $\varepsilon_{4 \pi+l o s t \pi, \gamma}(E)$ is the detection efficiency when we take not only fully reconstructed events but also events with lost $\pi^{ \pm}$or $\gamma$. The error of the correction factor was treated as systematic uncertainty of the efficiency (Fig. 4).

To separate events of the intermediate state $\omega \pi^{0}$ from others, we used the invariant mass distribution of the system $\pi^{+} \pi^{-} \pi^{0}$ in the reconstruction $\pi^{+} \pi^{-} \pi^{0} \pi^{0}$ that is closest to the $\omega$-meson mass $\left(M_{3 \pi}\right)$ (Fig. 5). The separated cross section is shown at (Fig. 6).

\section{Conclusion}

In an experiment with Spherical Neutral Detector at VEPP-2000 collider the cross section of the process $e^{+} e^{-} \rightarrow \pi^{+} \pi^{-} \pi^{0} \pi^{0}$ was measured in the energy range $1 \mathrm{GeV}<\sqrt{s}<2 \mathrm{GeV}$. Cross section is in agreement with previous measurements but has the best accuracy to date; the statistical 


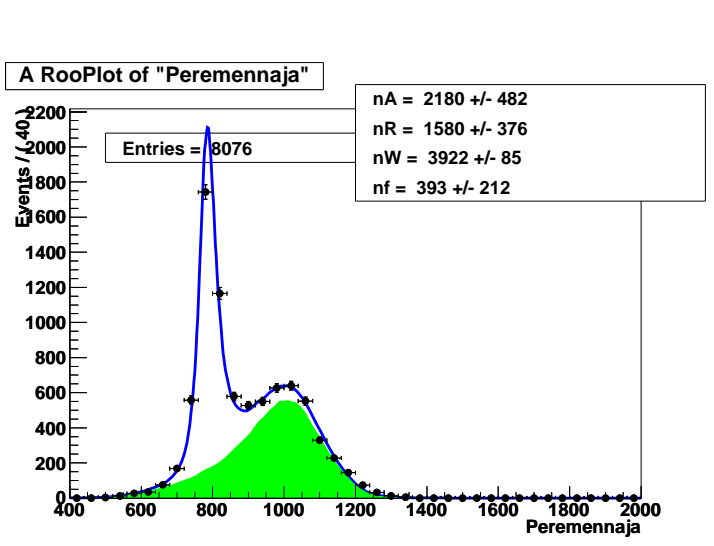

Figure 5: $\pi^{ \pm} \pi^{0} \pi^{0}$ invariant mass distribution.

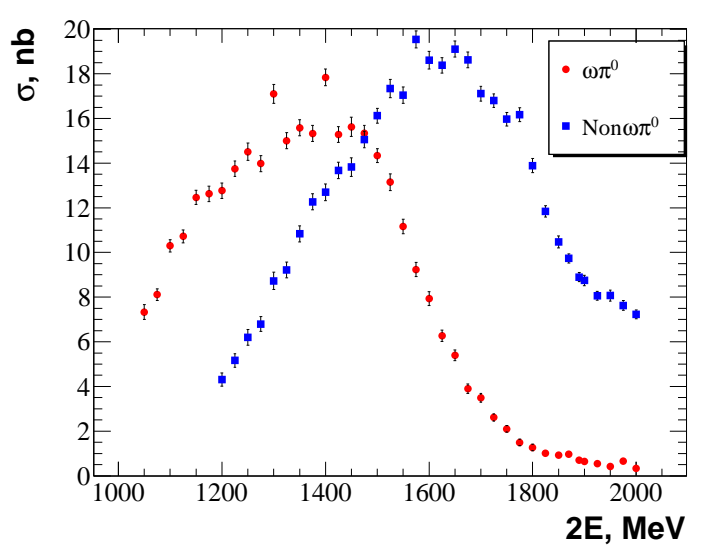

Figure 6: Cross section for the $\omega \pi^{0}$ and non- $\omega \pi^{0}$ sub process.

error is $1-2 \%$ the systematic uncertainty is $1.6-1.8 \%$ and we are working to improve it. The cross section corresponding to $\omega \pi^{0}$ intermediate state was separated from other states.

\section{Acknowledgments}

This work is supported by the Ministry of Education and Science of the Russian Federation (Contract 14.518.11.7003), the Russian Federation Presidential Grant for Scientific Schools NSh5320.2012.2, RFBR (grants 11-02-00276-a, 12-02-00065-a, 13-02-00418-a, 13-02-00375-a 12-0231488-mol-a, 12-02-31692-mol-a, 12-02-31488-mol-a, 12-02-33140-mol-a-ved), the Russian Federation Presidential Grant for Young Scientists MK-4345.2012.2 and the Grant 14.740.11.1167 from the Federal Program Scientific and Pedagogical Personnel of Innovational Russia”.

\section{References}

[1] M. N. Achasov, V. M. Aulchenko, S. E. Baru, et al, Nucl. Instrum. Methods Phys. Res., Sect. A 449, 125 (2000).

[2] A. N. Skrinsky, in Proc. of the Workshop on Physics and Detectors for DAPHNE, Frascati, Italy, 1995 (Frascati, 1995), p. 3. 